\title{
String loop corrections and de Sitter vacua
}

\author{
Ignatios Antoniadis ${ }^{1,2}$, Yifan Chen $^{3}$, George K. Leontaris ${ }^{* 4}$ \\ ${ }^{1}$ LPTHE Sorbonne Université, CNRS, 4 Place Jussieu, 75005 Paris, France \\ E-mail: antoniad@lpthe.jussieu.fr \\ 2 ITP, Albert Einstein Center, University of Bern, Sidlerstrasse 5, CH-3012 Bern, Switzerland \\ ${ }^{3}$ CAS Key Laboratory of Theoretical Physics, Insitute of Theoretical Physics, Chinese Academy \\ of Sciences, Beijing 100190, P.R.China \\ E-mail: yifan.chen@itp.ac.cn \\ ${ }^{4}$ Theory Section, Physics Department, University of Ioannina, GR-45110 Ioannina, Greece \\ E-mail: leonta@uoi.gr
}

The problem of moduli stabilisation is discussed in type IIB/F-theory. Considering a configuration of three intersecting D7 branes with fluxes, it is shown that higher loop effects induce logarithmic corrections to the Kähler potential which can stabilise Kähler moduli, in particular the internal volume of the compactification space in 4 dimensions. It is also explained how Dterm contributions to the effective potential stabilise the rest of the Kähler moduli and uplift to de Sitter vacua.

Corfu Summer Institute 2019 "School and Workshops on Elementary Particle Physics and Gravity" (CORFU2019)

31 August - 25 September 2019

Corfu, Greece

${ }^{*}$ Speaker. 


\section{Introduction}

Nowadays there is adversarial debate amongst high energy physics community whether the string theory landscape contains de Sitter (dS) vacua. No go theorems such as [1], and swampland conjectures [2] cast doubts on their existence in consistent quantum theories ${ }^{1}$ and disfavour cosmological inflation in effective field theory models of string origin. Instead, there are suggestions [4] that quintessence models where the cosmological constant varies over time satisfy current observational constraints. If so, the present acceleration phase shall come to an end and eventually the rapid expansion of the universe will terminate in the distant future. However, string quantum corrections which are anticipated to play essential role in shaping the final form of the effective theory and the scalar potential, have not been fully explored yet. Therefore, before abandoning the attractive de Sitter solutions which abide with successful inflationary models, it is worth exploring further the 'landscape' of string compactifications and the implications of various localised defects (such as D-branes) on the emerging effective theories. This presentation will focus on the investigation of dS vacua in type IIB superstring theory in the presence of D-branes.

There are several ways of building de Sitter vacua in the literature. Most common are those based on non-perturbative corrections, such as the KKLT mechanism [5] and the so-called large volume scenario [6]. These constructions are much debadable however, due to the fact that they rely on non-perturbative effects which cannot be controlled at the full string level (some improvements of the original models have appeared using the nilpotent chiral multiplets [7], leading to a new mechanism of uplifting the vacua in the stringy landscape [8]).

In this work, de Sitter vacua will be sought only from sources inducing perturbative corrections. It will be argued that higher derivative terms in the ten-dimensional string effective action generate upon compactification a four-dimensional Einstein-Hilbert term localised in the internal space, which in turn induces logarithmic corrections to the scalar potential via loop effects. At this point, it is worth emphasising that such corrections are standard in the presence of D7-branes; they appear as quantum corrections either in the world-sheet or in the string coupling perturbation theory and have also been studied in different contexts in the past [9, 10]. Such contributions, break the no-scale structure of the Kähler potential and lead to an effective theory with some Kähler moduli stabilised, such as the internal six-dimensional volume [11]. On the other hand, D-term contributions associated with abelian symmetries which are present for instance in configurations of intersecting D7-branes, work as an "uplifting" mechanism, stabilising the remaining Kähler moduli and ensuring the existence of de Sitter vacua [11].

\section{Prelude}

The aim of this work is to propose a solution to the moduli stabilisation problem in II-B string theory and to examine whether a dS minimum exists in consistent theories (i.e. those having an ultraviolet (UV) completion), based only on perturbative corrections. To set the notation and focus

\footnotetext{
${ }^{1}$ For a review and further references see [3]
} 
only on what is needed from type II-B framework, the moduli fields to be used in the subsequent analysis are briefly presented. ${ }^{2}$

From the NS-NS sector, the relevant ingredients are the dilaton and KB-field:

$$
g_{\mu v}, \phi, B_{\mu v} \rightarrow B_{2}
$$

From the R-R sector, we make use of the various p-form potentials:

$$
C_{0}, C_{\mu \nu}, C_{\kappa \lambda \mu \nu} \rightarrow C_{p}, \quad ; \quad p=0,2,4 .
$$

The $C_{0}$ potential and the dilaton field, are used in the usual axion-dilaton combination denoted here with $S$ :

$$
S=C_{0}+i e^{-\phi} \rightarrow C_{0}+\frac{i}{g_{s}},
$$

where $g_{s}$ is the string coupling. In addition, we will consider a six-dimensional compactification on a CY threefold and denote the complex structure (CS) moduli with $z_{a}$, and the Kähler class moduli with $T_{i}$ :

$$
\text { CS : } z_{a}, a=1,2,3 \ldots h_{(2,1)}, \quad ; \quad \text { Kähler : } T_{i}, i=1,2,3, \ldots h_{(1,1)} .
$$

Note also that CS moduli are $(2,1)$-forms whilst Kähler moduli are $(1,1)$-forms, with their respective number $h_{(2,1)}$ and $h_{(1,1)}$. Obviously, if moduli remain massless at low energies, they give rise to fifth forces and create other cosmological problems in the effective field theory. Hence, the task is to generate a potential and assure positive mass-squared for all moduli fields.

The subsequent analysis will be presented in the framework of type II-B $N=1$ effective supergravity. In this context, the two basic ingredients related to the present work are the superpotential of the moduli fields and the Kähler potential. Type II-B compactifications on six-dimensional CY manifolds give rise to $N=2$ supersymmetry in four dimensions. This is broken to $N=1$ in the presence of 3-form fluxes and orientifold planes satisfying a set of conditions (see e.g. [12]). To construct the superpotential we need the field strengths derived from the KB field and the $p$-form potentials. We also need to introduce the holomorphic $(3,0)$-form $\Omega$ which is a function of the CS moduli

$$
F_{p}:=d C_{p-1}, \quad H_{3}:=d B_{2} \quad \Rightarrow \quad \mathbf{G}_{\mathbf{3}}:=F_{3}-S H_{3}, \quad \Omega=\Omega\left(z_{a}\right) .
$$

Then, the fluxed induced superpotential $\mathscr{W}_{0}$, at the classical level, is given by the well-known formula [13]:

$$
\mathscr{W}_{0}=\int \mathbf{G}_{\mathbf{3}} \wedge \Omega\left(z_{a}\right)
$$

Clearly, the perturbative superpotential $\mathscr{W}_{0}$ is a holomorphic function and depends on the axiondilaton modulus $S$, and the CS moduli $z_{a}$. Thus, we apply the supersymmetric conditions, w.r.t. these fields $z_{a}, S$ by setting the Kähler covariant derivatives to zero:

$$
\mathscr{D}_{z_{a}} \mathscr{W}_{0}=0 \quad, \quad \mathscr{D}_{S} \mathscr{W}_{0}=0 .
$$

\footnotetext{
${ }^{2}$ The usual abbreviations for Calabi-Yau (CY), Neveu-Schwarz (NS), Ramond (R), and Kalb-Ramobd (KB) are also used.
} 
The above conditions fix the moduli $z_{a}, S$ which are present in the superpotential $\mathscr{W}_{0}$. On the contrary, the Kähler moduli, being $(1,1)$-forms, do not enter in the superpotential and thus, they remain completely undetermined at this stage. Nevertheless, we may appeal to the second ingredient of the effective supergravity which is the Kähler potential that depends logarithmically on the various moduli fields, including the Kähler moduli:

$$
\begin{aligned}
\mathscr{K}_{0} & =-2 \ln (i \mathscr{V})-\ln \left(-i \int \Omega \wedge \bar{\Omega}\right) \\
& =-\sum_{i=1}^{3} \ln \left(-i\left(T_{i}-\bar{T}_{i}\right)\right)-\ln (-i(S-\bar{S}))-\ln \left(-i \int \Omega \wedge \bar{\Omega}\right),
\end{aligned}
$$

where $\mathscr{V}$ is the volume of the $\mathrm{CY}$ depending on the Kähler class moduli. We then specialised for simplicity to the case of the three geometric moduli of the six dimensional torus $T^{6}$ corresponding the areas of three orthogonal planes $T^{2} \times T^{2} \times T^{2}$. One can now compute the effective potential using the standard formula

$$
V_{\text {eff }}=e^{\mathscr{K}}\left(\sum_{I, J} \mathscr{D}_{I} \mathscr{W}_{0} \mathscr{K}^{I \bar{J}} \mathscr{D}_{\bar{J}} \mathscr{W}_{0}-3\left|\mathscr{W}_{0}\right|^{2}\right)
$$

This can be split it in two parts:

$$
\begin{aligned}
V_{\mathrm{eff}}= & e^{\mathscr{K}} \sum_{\{I, J\}=\left\{z_{a}, S\right\}} D_{I} \mathscr{W}_{0} \mathscr{K}_{I \bar{J}}^{-1} D_{\bar{J}} \mathscr{W}_{0} \quad\left(D_{I} \mathscr{W}_{0}=0, \text { supersymmetry }\right) \\
& +e^{\mathscr{K}}\left(\sum_{i, j} \mathscr{K}_{0}^{i \bar{j}} D_{i} \mathscr{W}_{0} D_{\bar{j}} \mathscr{W}_{0}-3\left|\mathscr{W}_{0}\right|^{2}\right) \quad(=0, \text { no scale structure }) \\
= & 0
\end{aligned}
$$

The first part of the scalar potential (2.5) involves the moduli fields $z_{a}, S$ and it vanishes due to the supersymmetric conditions (2.2). The second part (2.6) involves only the Kähler moduli $T_{i}$ and is also identically zero because of the no-scale structure of the Kähler potential (2.3). Hence, it is impossible to fix the Kähler moduli at this (classical) level.

From the above discussion, it is inferred that in order to stabilise the Kähler moduli it is necessary to go beyond the classical level and include higher order effects. In fact, when quantum corrections are included they break the no-scale structure of the Kahler potential. To this end, let us first assume a generic test function depending only on a single modulus $\tau$, representing such corrections in the form $=\gamma f(\tau)$, with $\gamma$ a small parameter measuring their strength. This breaks the no-scale structure and the Kähler potential takes the form in the large volume expansion ${ }^{3}$

$$
\mathscr{K}=-2 \log \left(\tau^{\frac{3}{2}}+\gamma f(\tau)\right), \mathscr{V}=\tau^{\frac{3}{2}},|\gamma|<1
$$

Expanding in $\gamma$, the F-term potential becomes

$$
V_{F} \propto \gamma \frac{3 f(\tau)-4 \tau f^{\prime}(\tau)+4 \tau^{2} f^{\prime \prime}(\tau)}{\tau^{\frac{9}{2}}} .
$$

As expected, when $\gamma \rightarrow 0$, the no-scale structure of the Kähler potential is restored and $V_{F} \equiv 0$.

\footnotetext{
${ }^{3} \tau$ is assumed to be a 4-cycle modulus and thus, the internal volume has the simple form $\mathscr{V}=\tau^{\frac{3}{2}}$.
} 
Next, let us consider the typical case of perturbation theory at large volume with a power-law in the modulus $\tau$, i.e., $f(\tau) \propto \tau^{n}$. In this case, the resulting scalar potential exhibits a monotonic behaviour in $\tau, V_{F} \propto \tau^{n-\frac{9}{2}}$, hence there is no minimum. On the other hand, if the function $f(\tau)$ is a logarithm of the modulus $\tau$, the potential contains two different types of terms competing each other and in principle a minimum can exist. Indeed:

$$
V_{F} \propto \gamma \tau^{n-\frac{9}{2}}\left(\log (\tau)-\frac{8}{3}\right)+\cdots \Rightarrow \exists\left(V_{F}\right)_{\min } \text { if } \gamma<0,
$$

i.e., at a certain value of $\tau$, the potential has a minimum provided $\gamma<0$. Note though that this is an anti-de Sitter (AdS) vacuum and, as we will see below, additional uplifting terms will be required to obtain the desired dS minimum. Furthermore, assuming an exrta constant correction $\xi$ in the Kähler potential:

$$
\mathscr{K}=-2 \log \left(\tau^{\frac{3}{2}}+\xi+\gamma \ln (\tau)\right),
$$

it is is found that the size of the volume at the minimum is controlled by the constant parameter $\mu \equiv e^{\xi / \gamma}$.

$$
\mathscr{V}_{\min }=\frac{e^{13 / 3}}{\mu} \quad ; \mu=e^{\xi / \gamma}
$$

The constant $\mu$ can be chosen so that we can have an exponentially large volume.

From the above simple example, it is obvious that the existence of a minimum depends crucially on the properties of the function $f(\tau)$ associated with the quantum corrections. Hence, the crux of the matter is whether a suitable type of quantum corrections exist in string theory.

\section{3. "Localised" Einstein-Hilbert term}

In this part of the presentation, the role of perturbative string loop corrections will be examined. Firstly, we recall the well known world-sheet corrections, perturbative in the string slope $\alpha^{\prime}$, which are found to be proportional to the Euler characteristic $\chi$ of the compactification manifold. Secondly, subsequent string loop contributions will be computed which are found to be very important in the presence of D7-branes.

Starting with $\alpha^{\prime}$ corrections, it was shown that in the large volume limit they imply a redefinition of the dilaton field: [12]

$$
e^{-2 \phi_{4}}=e^{-2 \phi_{10}}(\mathscr{V}+\xi)=e^{-\frac{1}{2} \phi_{10}}(\hat{\mathscr{V}}+\hat{\xi}),
$$

where $\phi_{D}$ is the $D$-dimensional dilaton ( $\phi_{10}$ is fixed from the supersymmetry condition (2.2)) and the last expression holds in the Einstein frame. The volume can be expressed in terms of the imaginary parts $t^{k}$ of the Kähler deformations $T^{k}$ as follows:

$$
\mathscr{V}=\frac{1}{3 !} \kappa_{i j k} t^{i} t^{j} t^{k}, t^{k}=-\operatorname{Im}\left(T^{k}\right)=\hat{t}^{k} e^{\frac{1}{2} \phi_{10}} .
$$

Therefore, the corrections to the Kähler potential (2.3) can be accounted by a shift of the volume by a constant $\xi$ which is determined in terms of the Euler characteristic

$$
\xi=-\frac{\zeta(3)}{4(2 \pi)^{3}} \chi .
$$


The above corrections are associated with higher derivatives induced in the 10-dimensional effective action. Indeed, as is well known, multigraviton scattering in string theory generates higher derivative couplings. The leading terms in the effective action are those with eight derivatives and are proportional to $R^{4}$, (i.e., the Riemann tensor to the power four with appropriate contractions of indices). Upon compactification to four dimensions, they induce an Einstein-Hilbert (EH) term which is multiplied by the Euler characteristic of the manifold.

Indeed, let us just cast a glance back to the previous works incorporating the $R^{4}$ term in the Type II-B 10-dimensional effective action. According to [14, 15, 16, 12] the latter is given by

$$
\mathscr{S} \supset \frac{1}{(2 \pi)^{7} \alpha^{\prime 4}} \int_{M_{10}} e^{-2 \phi_{10}} \mathscr{R}_{(10)}-\frac{6}{(2 \pi)^{7} \alpha^{\prime}} \int_{M_{10}}\left(-2 \zeta(3) e^{-2 \phi_{10}}-4 \zeta(2)\right) R^{4} \wedge e^{2},
$$

where $\mathscr{R}_{(D)}$ is the $D$-dimensional Ricci scalar. Compactifying six dimensions on a CY manifold $\mathscr{X}_{6}$ (so that $M_{10}=M_{4} \times \mathscr{X}_{6}$ ), and taking into account the tree-level and the one-loop generated EH terms, the ten-dimensional action reduces to

$$
\mathscr{S}_{\text {grav }}=\frac{1}{(2 \pi)^{7} \alpha^{4}} \int_{M_{4} \times \mathscr{X}_{6}} e^{-2 \phi_{10} \mathscr{R}_{(10)}}+\frac{\chi}{(2 \pi)^{4} \alpha^{\prime}} \int_{M_{4}}\left(2 \zeta(3) e^{-2 \phi_{10}}+4 \zeta(2)\right) \mathscr{R}_{(4)},
$$

with the Euler characteristic defined as

$$
\chi=\frac{3}{4 \pi^{3}} \int_{\mathscr{X}_{6}} R \wedge R \wedge R .
$$

Therefore, after compactification, a localised EH term is generated with a coefficient proportional to the Euler characteristic $\chi$. From the above reduction and the form of $\chi$, it is also deduced that this effective EH-term is possible only in four dimensions.

More precisely, the above localisation is realised in the non-compact limit (large volume) at 'points' in the internal space where the Euler number $\chi$ is concentrated [16]. For instance, in the orbifold case, these are the fixed points of the orbifold group. At these points, there are localised vertices associated with the induced EH term, emitting gravitons and Kaluza-Klein (KK) excitations in the 6-dimensional space. A useful notion related to this mechanism is the localisation width which can be estimated by computing the graviton scattering involving two massless gravitons and one KK excitation (see Fig. 1). The computation can be done explicitly in the orbifold case, where the term proportional to $\zeta(3)$ in the action (3.4) vanishes and the induced EH-term arises at one loop order [16]. The result for the $T^{6} / Z_{N}$ orbifold is expressed as follows:

$$
\left\langle V_{(0,0)}^{2} V_{(-1,-1)}\right\rangle=-C_{R} \frac{1}{N^{2}} \sum_{f, k} e^{i \gamma^{k} q \cdot x_{f}} \int_{\mathscr{F}} \frac{d^{2} \tau}{\tau_{2}^{2}} \int \prod_{i=1,2,3} \frac{d^{2} z_{i}}{\tau_{2}} \sum_{(h, g)}{ }^{\prime} e^{\alpha^{\prime} q^{2} F_{(h, g)}\left(\tau, z_{i}\right)},
$$

where the subscripts in the vertices denote the ghost-picture, $q$ is the internal (KK) momentum, $C_{R}$ is a constant related to the tensor structure, whilst $x_{f}$ are the fixed points of the orbifold and $\gamma^{k}$ is the representation of the action of the orbifold group. The pairs $(h, g)$ label the orbifold sectors and the prime in the sum excludes the untwisted sector $(0,0)$. The integration over the modulus of the world-sheet torus $\tau=\tau_{1}+i \tau_{2}$ is restricted as usual in the fundamental domain $\mathscr{F}$ of the modular group. 


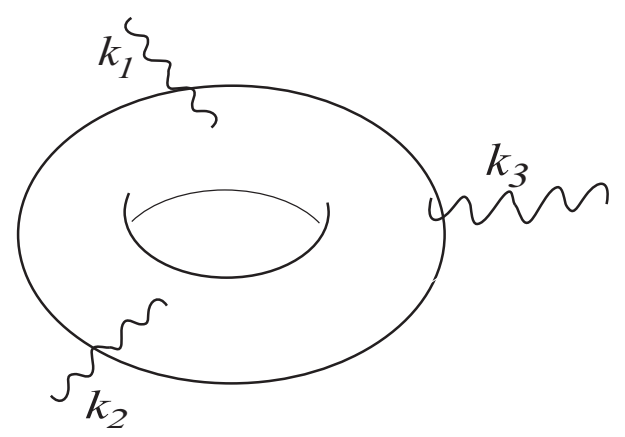

Figure 1: Non-zero contribution from 1-loop; 3-graviton scattering amplitude of 2 massless and $1 \mathrm{KK}$.

Referring to the relevant works for the details $[11,16]$, one finds that the width $w$ associated with the localised graviton vertices is given by

$$
\left.w^{2} \approx \alpha^{\prime} F_{(h, g)}\left(\tau, z_{a}\right)\right|_{\min } \sim \frac{l_{s}^{2}}{N}
$$

with $l_{s}=\sqrt{\alpha^{\prime}}$ the fundamental string length. The localisation property can be expressed in terms of the width (3.5), by computing the amplitude in the position space ' $y$ ', by a Fourier transform from the momentum space ' $q$ '. Indeed, it is found that the coefficient of the induced EH action $\mathscr{R}_{(4)}$ depending on the 6-dimensional internal space (in the position or momentum representation) exhibits a Gaussian profile

$$
C_{R} \frac{N}{w^{6}} e^{-w^{2} q_{\perp}^{2} / 2}
$$

controlled by the 'effective' width defined above. Here $q_{\perp}$ is the internal momentum along the directions allowed by momentum conservation. For instance, if the graviton emission ends on 7branes, $q_{\perp}$ is along the directions tranverse to their world-volume (see below). Then, the one loop correction takes the form

$$
\frac{4 \zeta(2)}{(2 \pi)^{7} \alpha^{\prime}} \chi \int_{M_{4} \times X_{6}} \frac{e^{-y^{2}} /\left(2 w^{2}\right)}{w^{6}} \mathscr{R}_{(4)}
$$

where $N$ has been interpreted as the Euler characteristic $\chi \sim N$.

In type II-B (as well as in F-theory) context, the geometric configuration may also include D7 branes and orientifold $\mathrm{O} 7$ planes. In effect, there can be an exchange of KK-modes between the localised gravity position (for instance an orbifold fixed-point) and the distinct 7-branes. Since D7 branes occupy four internal dimensions, KK-modes transmitted towards each one of them propagate in a 2-dimensional bulk transverse to D7 (see Fig. 2). In this way, logarithmic contributions are induced depending on the size of the 2-dimensional space transverse to D7. From the form of the effective EH term given in (3.4) it can be seen that this correction is multiplied by the Euler characteristic. Referring to the relevant works for the details $[11,16]$, the corresponding contribution takes the form

$$
\mathscr{A}_{\mathscr{S}}=-C_{R} g_{s}^{2} T \frac{2 \pi}{\sin \frac{2 \pi}{N}}\left\{-\frac{\gamma}{2}+\ln \left(\frac{R_{\perp} \sqrt{2}}{w}\right)+\mathscr{O}\left(\frac{w^{2}}{R_{\perp}^{2}}\right)\right\},
$$


whete $T$ is the $\mathrm{D} 7$ brane tension and $R_{\perp}$ measures the size of its transverse 2-dimensional space. In the above formula we are interested in the $R_{\perp}$ dependence, which exhibits a logarithmic behaviour. Summing up the two contributions (3.6) and (3.8), and replacing $\chi \sim N$ as above, one finds:

$$
\frac{4 \zeta(2)}{(2 \pi)^{3}} \chi \int_{M_{4}}\left(1-\sum_{k} e^{2 \phi} T_{k} \ln \left(R_{\perp}^{k} / w\right)\right) R_{(4)} .
$$

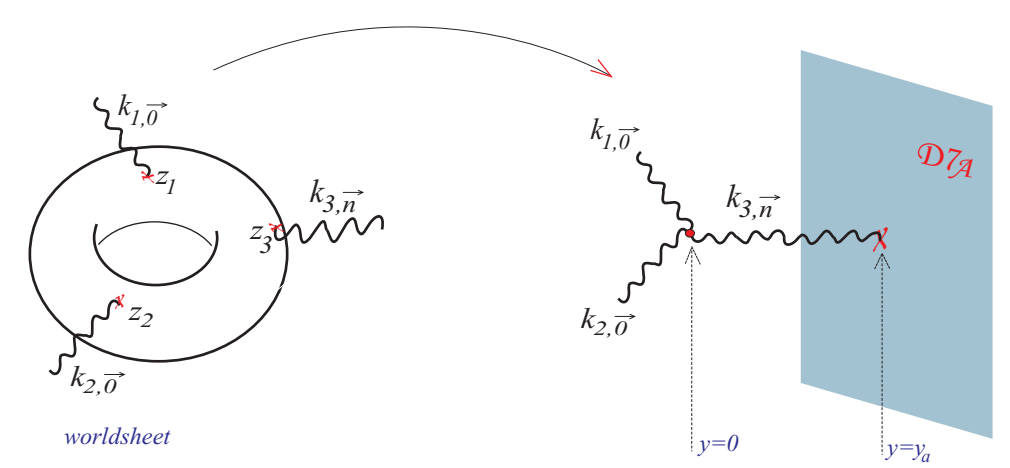

Figure 2: Non-zero contribution from 1-loop; 3-graviton scattering amplitude of 2 massless and 1 KK propagating in 2-dimensions towards a D7 brane.

\section{The effective potential}

The above corrections induce new terms to the Kähler potential. It can be shown [17] that stabilisation of the Kähler moduli requires at least three sets of D7 branes, with mutual orthogonal transverse spaces. Hence, the contributions involving the Kähler moduli are written in the form:

$$
\delta=\sum_{k=1}^{3} \gamma_{k} \ln \left(\tau_{k}\right)
$$

For simplicity, we take $\gamma_{k}=\gamma / 2$. Then, the Kähler potential takes the form

$$
\mathscr{K}=-2 \ln \left(\sqrt{\tau_{1} \tau_{2} \tau_{3}}+\xi+\gamma \ln \left(\tau_{1} \tau_{2} \tau_{3}\right)\right) \equiv-2 \ln (\mathscr{V}+\xi+\gamma \ln \mathscr{V})
$$

It can be easily checked that these corrections do break the no-scale structure. The F-term scalar potential is computed using (4.1). Expanding in $\gamma$, it takes the form

$$
V_{F} \approx 3 \gamma w_{0}^{2} \frac{\ln \mu \mathscr{V}-4}{\mathscr{V}^{3}}+\mathscr{O}\left(\gamma^{3}\right)
$$

where $\xi$ has been substituted with $\gamma \ln \mu$, and $w_{0}=\left\langle\mathscr{W}_{0}\right\rangle$.

In the presence of D7 branes, it is also possible that D-terms appear. These give rise to a contribution of form

$$
V_{D}=\sum_{a=1}^{3} \frac{d_{a}}{\tau_{a}}\left(\frac{\partial \mathscr{K}}{\partial \tau_{a}}\right)^{2} \approx \sum_{a=1}^{3} \frac{d_{a}}{\tau_{a}^{3}}+\cdots
$$

with $d_{a}$ numerical constants. Thus, the effective potential is the sum

$$
V_{\mathrm{eff}}=V_{F}+V_{D}
$$


Minimisation with respect to the volume implies the condition [17]:

$$
\gamma w_{0}^{2}(13-3 \ln \mathscr{V})=2 d \mathscr{V} ; d=\left(d_{1} d_{2} d_{2}\right)^{\frac{1}{3}}
$$

The solution of the above equation determines the volume at the extrema and depends on the double-valued Lambert W-function[17], which takes real double values in the region between $-1 / e$ and 0 . Hence, in this region the potential has a maximum and a minimum. However, not all values of this region are acceptable since the requirement for de Sitter vacua puts additional constraints. Putting all these together, the following constraints apply to the various constants

$$
-0.007242<\rho<-0.006738, \rho=\frac{d}{\gamma w_{0}^{2} \mu}
$$

In Fig. 3 the effective potential $V_{\text {eff }}$ is plotted versus the volume $\mathscr{V}$ for fixed values of the parameter $\rho$. The values of $\rho$ in the upper two curves lies within the acceptable region (4.3) and hence they yield de Sitter minima. On the contrary, the lower curve is for a value of $\rho$ outside the above region and thus, it corresponds to an AdS vacuum. At large volume, the potential vanishes asymptotically after passing from a maximum.

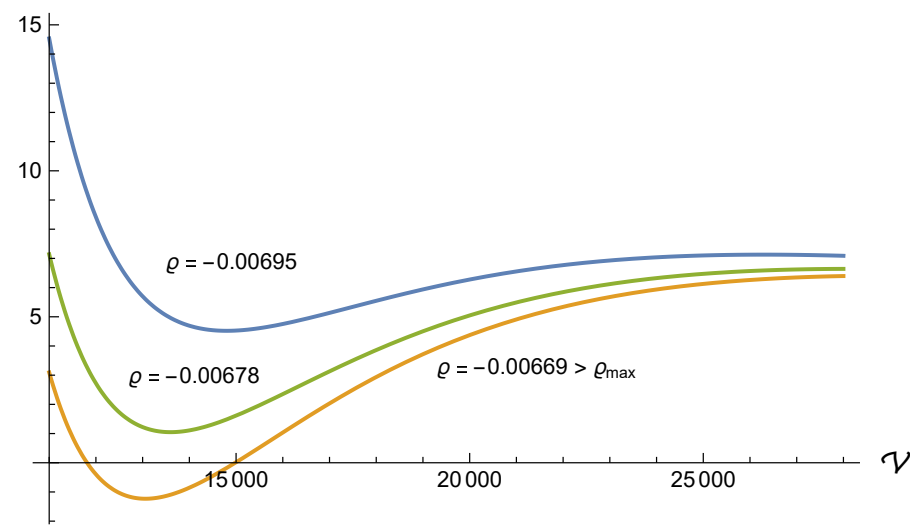

Figure 3: Plot of the scalar potential as a function of the volume for different values of the parameter $\rho$

\section{Conclusions}

In this presentation it has been demonstrated that perturbative corrections in a specific D7brane configuration of type II-B string compactifications have the appropriate ingredients to stabilise the Kähler moduli. The origin of these corrections come from the reduction of $R^{4}$ couplings of the ten-dimensional string effective action and can exist only in 4-dimensions [18]. One may conclude that this is an indispensable element for the existence of 4-dimensional de Sitter vacua.

\section{Acknowledgements}

Work was supported in part by the Labex "Institut Lagrange de Paris", in part by the Swiss National Science Foundation, in part by a CNRS PICS grant, and in part by the Erasmus exchange program. 


\section{References}

[1] J. M. Maldacena and C. Nunez, "Supergravity description of field theories on curved manifolds and a no go theorem,” Int. J. Mod. Phys. A 16 (2001) 822 [hep-th/0007018].

[2] C. Vafa, "The String landscape and the swampland," hep-th/0509212.

G. Obied, H. Ooguri, L. Spodyneiko and C. Vafa, "De Sitter Space and the Swampland," arXiv:1806.08362 [hep-th].

[3] E. Palti, “The Swampland: Introduction and Review," Fortsch. Phys. 67, no. 6, 1900037 (2019) [arXiv:1903.06239 [hep-th]].

[4] P. Agrawal, G. Obied, P. J. Steinhardt and C. Vafa, "On the Cosmological Implications of the String Swampland,” Phys. Lett. B 784 (2018) 271 [arXiv:1806.09718 [hep-th]].

[5] S. Kachru, R. Kallosh, A. D. Linde and S. P. Trivedi, "De Sitter vacua in string theory,” Phys. Rev. D 68 (2003) 046005 [hep-th/0301240].

[6] J. P. Conlon, F. Quevedo and K. Suruliz, "Large-volume flux compactifications: Moduli spectrum and D3/D7 soft supersymmetry breaking," JHEP 0508 (2005) 007 [hep-th/0505076].

[7] I. Antoniadis, E. Dudas, S. Ferrara and A. Sagnotti, “The Volkov?Akulov?Starobinsky supergravity," Phys. Lett. B 733 (2014) 32 [arXiv:1403.3269 [hep-th]].

[8] S. Ferrara, R. Kallosh and A. Linde, “Cosmology with Nilpotent Superfields,” JHEP 1410 (2014) 143 [arXiv:1408.4096 [hep-th]].

[9] I. Antoniadis and C. Bachas, "Branes and the gauge hierarchy," Phys. Lett. B 450, 83 (1999) [hep-th/9812093].

[10] W. D. Goldberger and M. B. Wise, "Renormalization group flows for brane couplings," Phys. Rev. D 65 (2002) 025011 [hep-th/0104170].

[11] I. Antoniadis, Y. Chen and G. K. Leontaris, "Logarithmic loop corrections, moduli stabilisation and de Sitter vacua in string theory,” JHEP 2001 (2020) 149 [arXiv:1909.10525 [hep-th]].

[12] K. Becker, M. Becker, M. Haack and J. Louis, "Supersymmetry breaking and alpha-prime corrections to flux induced potentials," JHEP 0206 (2002) 060 [hep-th/0204254].

[13] S. Gukov, C. Vafa and E. Witten, Nucl. Phys. B 584 (2000), 69-108 doi:10.1016/S0550-3213(00)00373-4 [arXiv:hep-th/9906070 [hep-th]].

[14] I. Antoniadis, S. Ferrara, R. Minasian and K. S. Narain, " $R^{4}$ couplings in M and type II theories on Calabi-Yau spaces,” Nucl. Phys. B 507 (1997) 571 [hep-th/9707013].

[15] E. Kiritsis and B. Pioline, "On $R^{4}$ threshold corrections in IIb string theory and (p, q) string instantons,” Nucl. Phys. B 508, 509 (1997) [hep-th/9707018].

[16] I. Antoniadis, R. Minasian and P. Vanhove, "Noncompact Calabi-Yau manifolds and localized gravity,” Nucl. Phys. B 648 (2003) 69 [hep-th/0209030].

[17] I. Antoniadis, Y. Chen and G. K. Leontaris, "Perturbative moduli stabilisation in type IIB/F-theory framework,” Eur. Phys. J. C 78 (2018) no.9, 766 [arXiv:1803.08941 [hep-th]].

[18] I. Antoniadis, R. Minasian, S. Theisen and P. Vanhove, "String loop corrections to the universal hypermultiplet," Class. Quant. Grav. 20 (2003) 5079 [hep-th/0307268]. 